\title{
Sero-surveillance of Transmissible Gastroenteritis Virus (TGEV) and Porcine Respiratory Coronavirus (PRCV) in South Korea
}

\author{
Yoon-I Oh${ }^{*}$, Dong-Kun Yang, Soo-Dong Cho, Hee-Kyung Kang, Sun-Keum Choi, \\ Ye-Jee Kim, Bang-Hun Hyun and Jae-Young Song \\ Animal, Plant and Fisheries Quarantine and Inspection Agency, Anyang, 430-757, Korea \\ MIFAFF, Korea
}

\begin{abstract}
Transmissible gastroenteritis (TGE) is sporadic in South Korea. Since porcine respiratory coronavirus (PRCV) infection was identified in South Korea in 1996, the TGE infection has decreased with the PRCV occurrence. In this study, we described the sero-surveillance of TGE/PRCV infection by using a commercially available ELISA kit. A total of 1,295 sera from slaughtered pigs and 69 sera from wild boars were collected in years 2009 and 2010 throughout the country and tested for antibodies against TGE and PRCV. Although there was no clinical sign observed for TGE and vaccination had not been done for TGEV, $4.9 \%$ of sera showed positive for antibody against TGEV. Furthermore, $63.7 \%$ of finisher and $8.7 \%$ of wild boars were positive for antibody against PRCV. Our result suggests that the TGEV infection might still be present in some farms in South Korea.
\end{abstract}

Key Words: TGE, PRCV, Differentiation of porcine coronavirus, Surveillance

\section{INTRODUCTION}

Transmissible gastroenteritis (TGE) is a highly contagious enteric disease of pigs caused by the TGE virus (TGEV), a member of the Coronaviridae. During an outbreak of TGE in a naïve swine herd, the TGEV spreads rapidly to pigs of all ages and causes vomiting, watery diarrhoea, dehydration, and death, particularly in pigs under 2 weeks of age (1).

A distinct respiratory variant, porcine respiratory coronavirus (PRCV) has spread throughout the world since 1984 (2 4). PRCV is considered as a mutant of TGEV with a

Received: August 20, 2011/ Revised: August 25, 2011

Accepted: August 30, 2011

* Corresponding author: Yoon-I Oh. Animal, Plant and Fisheries Quarantine and Inspection Agency, Anyang 480, Manan-gu, Anyang, 430-757, Korea. Phone: +82-31-467-1784, Fax: +82-31-467-1797

e-mail: yoonioh@korea.kr

** This work was supported financially by a grant from the Animal, Plant and Fisheries Quarantine and Inspection Agency (formerly National Veterinary Research and Quarantine Service), Ministry for Food, Agriculture, Forestry and Fisheries, Republic of Korea. characteristic large deletion in the 5 ' region of the spike (S) protein gene, which leads to a loss of an antigenic determinant $(3,5,6)$. PRCV, a deletion mutant of TGEV, showed two amino acids depletion from TGEV $S$ protein resulting in a change from gastrointestinal to respiratory tropism (7).

TGEV multiplies in and damages the enterocytes lining the small intestine, producing villous atrophy and enteritis. The virus is mostly isolated from the intestinal tract and from faeces. By contrast, PRCV is mostly isolated from the upper respiratory tract, trachea, tonsils or the lungs.

PRCV itself does not appear to be an important primary pathogen, but it contributes to the porcine respiratory disease complex. Furthermore, the serological diagnosis of TGEV is greatly complicated since both TGEV and PRCV induce similar level of neutralizing antibody titres. Therefore, the differential diagnosis of TGEV and PRCV infection can only be accomplished by a blocking ELISA (8).

The purpose of this study was to investigate the prevalence of TGEV and PRCV antibodies throughout the country. In 
this study, a commercially available blocking ELISA kit was used to differentiate between TGEV and PRCV infection from the sera of slaughtered pigs and wild boars throughout the country.

\section{MATERIALS AND METHODS}

\section{Samples from finisher}

Serum samples were collected from the finisher before they were slaughtered in year 2009. These serum samples were taken from the annual surveillance programme for classical swine fever (CSF).

\section{Samples from wild boars}

Wild boars were captured and serum samples were taken. Serum samples were collected from 5 provinces, i.e., Gangwon, Gyeongnam, Jeonnam, Jeonbuk, and Chungbuk. All samples were taken between November 2009 and February 2010.

\section{Serological test}

An Ingezim Corona Differential ELISA kit (Ingenasa, Spain) was used for the specific detection and differentiation of antibodies against TGEV and PRCV in pig serum. The kit applied a recombinant antigen and a couple of specific monoclonal antibodies: one to recognize generic epitopes to TGEV and PRCV and the other to recognize a specific epitope from TGEV. The ELISA was carried out according to the manufacturer's instructions.

Briefly, samples and kits were allowed to room temperature before use. Fifty microlitres of each serum and serum diluent were added in duplicates to the appropriate wells, and then plates were incubated for 1 hour at $37^{\circ} \mathrm{C}$. The plates were washed three times with washing solution. A hundred microlitres of conjugates were added to each well: conjugate A to one of the wells of each sample then conjugate $\mathrm{B}$ to the second well of each sample. Then the plates were incubated for 30 minutes at $37^{\circ} \mathrm{C}$. The plates were washed 6 times and $100 \mu$ of substrate were added to each well then kept for 10 minutes at room temperature. The reactions were stopped by adding $100 \mu \mathrm{l}$ of stop
Table 1. List of serum samples taken throughout the country

\begin{tabular}{lcc}
\hline \hline Province & $\begin{array}{c}\text { No. of finisher } \\
(\%)\end{array}$ & $\begin{array}{c}\text { No. of wild boars } \\
(\%)\end{array}$ \\
\hline Gangwon & $102(7.9)$ & $30(43.5)$ \\
Gyeonggi & $291(22.5)$ & - \\
Gyeongnam & $142(11.0)$ & $3(4.3)$ \\
Gyeongbuk & $216(16.7)$ & - \\
Jeonnam & $70(5.4)$ & $13(18.8)$ \\
Jeonbuk & $106(8.2)$ & $15(21.7)$ \\
Jeju & $96(7.4)$ & - \\
Chungnam & $198(15.3)$ & - \\
Chungbuk & $74(5.7)$ & $8(11.6)$ \\
\hline Total & $1,295(100)$ & $69(100)$ \\
\hline
\end{tabular}

solution and the plates were read at $450 \mathrm{~nm}$.

\section{RESULTS}

\section{Serum samples from finisher}

A total of 1,295 sera from 102 farms were collected throughout the country in 2009 (Table 1). From Gangwon province, 102 serum samples were selected from three regions: Chuncheon, Hongcheon, and Hoengseong. From Gyeonggi province, 291 serum samples were selected from 6 regions: Anseong, Yangpyeong, Yeoju, Icheon, Pyeongtaek, and Pocheon. From Gyeongnam province, 142 serum samples were selected from 8 regions: Gyeongju, Gimhae, Masan, Miryang, Uiryeong, Jinju, Changwon, and Haman. From Gyeongbuk province, 216 serum samples were selected from 8 regions: Gumi, Gunwi, Gimcheon, Daegu, Seongju, Andong, Yeongcheon, and Pohang. From Jeonnam province, 70 serum samples were selected from four regions: Goheung, Gwangyang, Boseong, and Suncheon. From Jeonbuk province, 106 serum samples were selected from three regions: Gunsan, Gimje, and Jeongeup. From Jeju Special Self-governing Province, 96 serum samples were selected from 5 regions: Seogwipo, Aewol, Jeju, Jocheon, and Hallim. From Chungnam province, 198 serum samples were selected from 7 regions: Dangjin, Buyeo, Asan, Yesan, Cheonan, Cheongyang, and Hongseong. From Chungbuk 
Table 2. Result of antibody test for TGEV and PRCV in finisher and wild boars

\begin{tabular}{|c|c|c|c|c|}
\hline \multirow{2}{*}{ Province } & \multicolumn{2}{|c|}{ Finisher (\% Seropositive) } & \multicolumn{2}{|c|}{ Wild boars (\% Seropositive) } \\
\hline & TGEV antibody & PRCV antibody & TGEV antibody & PRCV antibody \\
\hline Gangwon & $0(0 / 102)^{\mathrm{a}}$ & $18.6(19 / 102)$ & $0(0 / 30)$ & $20(6 / 30)$ \\
\hline Gyeonggi & $3.1(9 / 291)$ & $70.4(205 / 291)$ & - & - \\
\hline Gyeongnam & $1.4(2 / 142)$ & $57.7(82 / 142)$ & $0(0 / 3)$ & $0(0 / 3)$ \\
\hline Gyeongbuk & $6.5(14 / 216)$ & $69.4(150 / 216)$ & - & - \\
\hline Jeonnam & $0(0 / 70)$ & $0(0 / 70)$ & $0(0 / 13)$ & $0(0 / 13)$ \\
\hline Jeonbuk & $1.9(2 / 106)$ & $92.5(98 / 106)$ & $0(0 / 15)$ & $0(0 / 15)$ \\
\hline Jeju & $9.4(9 / 96)$ & $75(72 / 96)$ & - & - \\
\hline Chungnam & $11.6(23 / 198)$ & $67.7(134 / 198)$ & - & - \\
\hline Chungbuk & $6.8(5 / 74)$ & $87.8(65 / 74)$ & $0(0 / 8)$ & $0(0 / 8)$ \\
\hline Total & $4.9(64 / 1295)$ & $63.7(825 / 1295)$ & $0(0 / 69)$ & $8.7(6 / 69)$ \\
\hline
\end{tabular}

a $\%$ positivity (No. of positive sera/No. of tested sera).

province, 74 serum samples were selected from three regions: Eumseong, Jincheon, and Cheongwon.

\section{Serum samples from wild boars}

A total of 69 sera of wild boars were collected from 5 provinces in South Korea from November 2009 to February 2010 (Table 1). From Gangwon province, 30 samples were taken. Three samples were taken from Gyeongnam province, 13 from Jeonnam, 15 from Jeonbuk province, and 8 from Chungbuk province, respectively.

\section{Porcine coronavirus in finisher}

A total of 1,295 finisher sera were collected from slaughtering houses in 2009 and tested for the detection and differentiation of antibodies against TGEV and PRCV (Table 2). Throughout the country, $68.6 \%$ (889 pigs) were found to be positive for porcine coronavirus. Out of 889 coronavirus positive sera, 64 pigs (4.9\%) were positive for TGEV and 825 pigs (63.7\%) were positive for PRCV. There was no porcine coronavirus-positive pig detected in Jeonnam province and no TGEV-positive pig detected in Gangwon province, although there were PRCV-positive pigs (19 out of 102 pigs). Overall, less than $10 \%$ of TGEV-positive pigs were found in South Korea except in Chungnam province $(11.6 \%)$. More than half of the pigs in South Korea were positive for PRCV except in Gangwon and Jeonnam provinces.

\section{Porcine coronavirus in wild boars}

Serum samples from wild boars were tested for the detection and differentiation of antibodies against TGEV and PRCV (Table 2). Out of total 69 samples, 6 (8.7\%) from Gangwon province were positive for PRCV. No TGEV-positive sample was found from captured wild boars.

\section{TGE outbreaks in South Korea}

A total of 8 TGE outbreaks were reported in South Korea from 2004 to 2010 (Table 3). Four outbreaks occurred in Jeonnam and Kyeongnam in 2006. Three cases were reported in Jeonbuk, Kyeongbuk, and Kyeongnam in 2007. In 2010, a TGE outbreak occurred in one farm in Chungnam province.

\section{DISCUSSION}

PRCV has become enzootic in many swine herds globally. In Europe, the emergence of PRCV has greatly reduced the incidence and severity of epizootic TGE $(3,7)$. It was suggested in a TGEV-PRCV cross-protection study that repeated subclinical PRCV infections increased the level of 
Table 3. TGE outbreaks reported in South Korea from 2004 to $2010\left(\right.$ AIMS $\left.^{\text {a }}\right)$

\begin{tabular}{|c|c|c|c|c|c|c|c|c|c|c|}
\hline \multirow{2}{*}{ Year } & \multicolumn{9}{|c|}{ Province } & \multirow{2}{*}{ Total } \\
\hline & $\mathrm{GG}^{\mathrm{b}}$ & GW & $\mathrm{CB}$ & $\mathrm{CN}$ & JB & $\mathrm{JN}$ & GB & GN & $\mathrm{JJ}$ & \\
\hline 2004 & - & - & - & - & - & - & - & - & - & - \\
\hline 2005 & - & - & - & - & - & - & - & - & - & - \\
\hline 2006 & - & - & - & - & - & $\begin{array}{l}203^{\mathrm{c}} \\
{[2]^{\mathrm{d}}}\end{array}$ & - & $\begin{array}{c}1,350 \\
{[2]}\end{array}$ & - & $\begin{array}{c}1,553 \\
{[4]}\end{array}$ \\
\hline 2007 & - & - & - & - & $\begin{array}{l}50 \\
{[1]}\end{array}$ & - & $\begin{array}{l}150 \\
{[1]}\end{array}$ & $\begin{array}{l}20 \\
{[1]}\end{array}$ & - & $\begin{array}{c}220 \\
{[3]}\end{array}$ \\
\hline 2008 & - & - & - & - & - & - & - & - & - & - \\
\hline 2009 & - & - & - & - & - & - & - & - & - & - \\
\hline 2010 & - & - & - & $\begin{array}{l}160 \\
{[1]}\end{array}$ & - & - & - & - & - & $\begin{array}{l}160 \\
{[1]}\end{array}$ \\
\hline Total & - & - & - & $\begin{array}{l}160 \\
{[1]}\end{array}$ & $\begin{array}{l}50 \\
{[1]}\end{array}$ & $\begin{array}{c}203 \\
{[2]}\end{array}$ & $\begin{array}{l}150 \\
{[1]}\end{array}$ & $\begin{array}{c}1,370 \\
{[3]}\end{array}$ & - & $\begin{array}{c}1,933 \\
{[8]}\end{array}$ \\
\hline
\end{tabular}

a AMIS; Animal Infectious disease data Management System by National Veterinary Research and Quarantine Service, Korea.

${ }^{\mathrm{b}} \mathrm{GG}$; Gyeonggi, GW; Gangwon, CB; Chungbuk, CN; Chungnam, JB; Jeonbuk, JN; Jeonnam, GB; Gyeongbuk, GN; Gyeongnam, JJ; Jeju.

${ }^{\mathrm{c}}$ No. of positive animal.

${ }^{\mathrm{d}}$ No. of positive farm.

immunoglobulin A (IgA) antibodies cross-reactive to TGEV in the milk of lactating sows $(7,9)$. The TGEV infection in piglets born to such sows was characterized by reduced severity of clinical disease (7). It seems that PRCV acts as a naturally modified-live vaccine to TGEV and induces active immunity in pregnant sows, which is passively transferred to suckling piglets $(7,9)$. In South Korea, the incidence of epidemic TGE is sporadic as shown in Table 3. A total of 8 outbreaks were reported from 2004 to 2010 in South Korea. The PRCV infection was first described in South Korea in 1996 (10). High sero-prevalence to PRCV (61\%) was reported in South Korea, suggesting an extensive distribution of the virus throughout the swine population $(7,11)$.

In this study, we investigated the prevalence of TGEV and PRCV antibodies from the sera collected in year 2009 throughout the country to determine whether the recent decreases in reported outbreaks of TGE is due to widespread of PRCV infection. The serum samples were collected as a part of annual surveillance programme for CSF. CSF, also known as hog cholera, is a contagious viral disease of pigs. Classical swine fever virus (CSFV) is a member of the genus Pestivirus of the family Flaviviridae. As a part of national prevention measures of epidemics, annual surveillance for antibodies against CSF is active in South Korea. The serum samples used in this study were kindly procured by the CSFV research group.

Though the prevalence varies among regions, the overall result showed that $4.9 \%$ of sera (64 out of 1,295 tested) from slaughterhouse pigs were TGEV positive, whereas $63.7 \%$ of sera ( 825 out of 1,295 tested) were PRCV positive (Table 2). Several experimental studies have reported that PRCV can induce a variable degree of protection against TGEV infection (12 16). As shown in Europe (7), the prevalence of TGEV infections decreased in coincidence with the spread of PRCV in South Korea. Antibodies against TGEV could be by TGEV infection not by vaccination since vaccination has rarely been done to finisher in South Korea. In this study, all the TGEV antibody-positive pigs were found at the PRCV-positive farms. PRCV itself is not an important primary pathogen but it has considerably complicated the diagnosis of TGE, particularly by serological means. In this study, the antibodies to PRCV were detected in 6 wild boars $(8.7 \%)$, whereas the antibodies to PRCV showed $64 \%$ positivity in domestic pigs. Therefore, transmission does not occur between two populations. This study only shows the sero-prevalence data from the year 
2009; therefore, further epidemiological study might be necessary to estimate the current situation of TGE outbreak in South Korea. Even though the sero-prevalence of TGEV is lower than $5 \%$, the TGE infection might be still present in some farms in South Korea. In summary, this paper shows the sero-prevalence of TGEV and PRCV in the Korean pig population. Throughout the country, PRCV is extensively distributed. The TGEV outbreak coincidentally reduced with the spread of PRCV. Even though the TGEV prevalence was reduced, the TGE infection sporadically occurs in Korea.

\section{REFERENCES}

1) Saif LJ, Wesley RD. Transmissible gastroenteritis and porcine respiratory coronavirus. In: Straw BE, D'Allaire S, Mengeling WL, Taylor DJ, editors. Diseases of Swine. 8th ed. Ames, Iowa USA: Iowa State University Press; 1999. p. 295-325.

2) Brown I, Cartwright S. New porcine coronavirus? Vet Rec 1986;119:282-3.

3) Laude H, Van Reeth K, Pensaert M. Porcine respiratory coronavirus: molecular features and virushost interactions. Vet Res 1993;24:125-50.

4) Pensaert M, Callebaut P, Vergote J. Isolation of a porcine respiratory, non-enteric coronavirus related to transmissible gastroenteritis. Vet Q 1986;8:257-61.

5) Vaughn EM, Halbur PG, Paul PS. Sequence comparison of porcine respiratory coronavirus isolates reveals heterogeneity in the S, 3, and 3-1 genes. J Virol 1995; 69:3176-84.

6) Rasschaert D, Duarte M, Laude H. Porcine respiratory coronavirus differs from transmissible gastroenteritis virus by a few genomic deletions. J Gen Virol 1990;71: 2599-607.

7) Sestak K, Saif LJ. Porcine coronaviruses. In: Morilla A, Yoon K-J, Zimmerman JJ, editors. Trends in Emerging Viral Infections of Swine. Ames, Iowa: Iowa State Press; 2002. p. 321-30.
8) Garwes DJ, Stewart F, Cartwright SF, Brown I. Differentiation of porcine coronavirus from transmissible gastroenteritis virus. Vet Rec 1988;122:86-7.

9) Sestak K, Lanza I, Park SK, Weilnau PA, Saif LJ. Contribution of passive immunity to porcine respiratory coronavirus to protection against transmissible gastroenteritis virus challenge exposure in suckling pigs. Am J Vet Res 1996;57:664-71.

10) Ahn K, Chae C, Kweon $\mathrm{CH}$. Immunohistochemical identification of porcine respiratory coronavirus antigen in the lung of conventional pigs. Vet Pathol 1997;34: 167-9.

11) Chae C, Kim O, Min K, Choi C, Kim J, Cho W. Seroprevalence of porcine respiratory coronavirus in selected Korean pigs. Prev Vet Med 2000;46:293-6.

12) Bernard S, Bottreau E, Aynaud JM, Have P, Szymansky J. Natural infection with the porcine respiratory coronavirus induces protective lactogenic immunity against transmissible gastroenteritis. Vet Microbiol 1989;21:1-8.

13) Cox E, Pensaert MB, Callebaut P. Intestinal protection against challenge with transmissible gastroenteritis virus of pigs immune after infection with the porcine respiratory coronavirus. Vaccine 1993;11:267-72.

14) Kim L, Hayes J, Lewis P, Parwani AV, Chang KO, Saif LJ. Molecular characterization and pathogenesis of transmissible gastroenteritis coronavirus (TGEV) and porcine respiratory coronavirus (PRCV) field isolates co-circulating in a swine herd. Arch Virol 2000;145: 1133-47.

15) Wesley RD, Lager KM. Increased litter survival rates, reduced clinical illness and better lactogenic immunity against TGEV in gilts that were primed as neonates with porcine respiratory coronavirus (PRCV). Vet Microbiol 2003;95:175-86.

16) Wesley RD, Woods RD. Immunization of pregnant gilts with PRCV induces lactogenic immunity for protection of nursing piglets from challenge with TGEV. Vet Microbiol 1993;38:31-40. 